\title{
Klebsiella pneumoniae KPC: first isolations in Italy
}

\author{
Carla Fontana ${ }^{1,2}$, Marco Favaro ', Loredana Sarmati ${ }^{3}$, Silvia Natoli ${ }^{4}$, Anna Altieri', Maria Cristina Bossa ${ }^{2}$, \\ Silvia Minelli ${ }^{2}$, Cartesio Favalli ${ }^{1,2}$ \\ ' Department of Experimental Medicine and Biochemical Science University of Tor Vegata Rome \\ ${ }^{2}$ Microbiology Lab, Polyclinic Tor Vergata Foundation \\ ${ }^{3}$ Infectiouse Disease, Polyclinic Tor Vergata Foundation \\ ${ }^{4} \mathrm{ICU}$, Polyclinic Tor Vergata Foundation
}

Key words: Klebsiella pneumoniae, PCR, KPC, Hodge's test

Klebsiella pneumoniae KPC: primi isolamenti in Italia

\section{SUMMARY}

Klebsiella pneumoniae carbapenemase (KPC) was detected in two isolates of carbapenem-resistant K. pneumoniae in an italian teaching hospital. This is the first report of a KPC-producing isolates in our country. The first strain was isolated from a urine sample collected from a indwelling urinary catheter in a ICU-patient with subdural haematoma, while the second was from the culture of the central venous catheter (CVC) in a patient affected by Crohn's disease admitted in gastroenterology ward. Both were resistant to all B-lactams, susceptible to imipen$\mathrm{em}$ and meropenem and resistant to ertapenem. They were resistant to other classes of non-ß-lactams antibiotics such as quinolones, aminoglycosides (with the exception of amikacin), trimethoprim-sulfamethoxazole (TMP-SMX) as well as to nitrofurantoin. The isolates were not associated with travel abroad. They were found to contain the plasmid encoded carbapenemase gene blakp and were also positive to the Hodge's test. The detection of KPC-producing bacteria has important implications in infection control and public health.

The K. pneumoniae carbapenemase (KPC) belong to class A B-lactamases of the functional group 2f. Reported for the first time in U.S. in $200 \mathrm{I}$, these agents were subsequently identified in Europe. KPC strains are typically resistant to penicillins, extended-spectrum cephalosporin and aztreonam and present a peculiar behavior against carbapenems in that MIC is close to the susceptibility value or is borderline (except for ertapenem). This pattern is often associated with resistance to quinolones. The information is conveyed by the resistance plasmids, thus explaining their diffusion and implication in outbreaks of KPC. Despite this, to date there are few reports concerning the isolation of this phenotype in Italy. The purpose of this paper is to present two clinical cases related to the isolation of KPC in our hospital.

The KPC-producing strains have been respectively isolated: the first in a patient with Crohn's disease, and the second in a patient with a subdural haematoma. Nono of these patient had a recent history of travel abroad. The strains were characterized by Vitek 2 and E-test (bioMérieux). The phenotype was confirmed through the execution of the Hodge's test and by PCR blaKPC. The genetic relationship between isolates was determined by Rep-PCR.

Both isolates were resistant to B-lactams, quinolones and ertapenem, and were susceptible to imipenem, meropenem, tigecycline and colistin. The Hodge's test was positive and the sequence analysis of the blaKPC gene revealed a KPC gene type 2. The fingerprinting showed that the isolates were clonally related. Both patients were successfully treated with tigecycline.

KPC represents a real challenge either for the clinicians, who have limited therapeutic options to treat infections due to this microorganism, and for the laboratory, since their correct identification is necessary to reduce the dissemination of the pathogen.

La K. pneumoniae carbapenemase (KPC) appartiene alle ßlattamasi di classe A (contenenti serina) che appartengono al gruppo funzionale $2 \mathrm{f}$. Segnalate per la prima volta in USA nel 2001(1) sono state identificate successivamente anche in Europa.

I ceppi KPC sono tipicamente resistenti alle penicilline, alle cefalosporine a spettro esteso ed all'aztreonam $(2,3)$ presentano un comportamento peculiare nei confronti dei carbapenemi: le MIC sono prossime alla sensibilità o borderline (eccezion fatta per ertapenem).

A questo pattern si associa spesso la resistenza ai chinolonici. L'informazione della resistenza è veicolata da plasmidi $(4,5)$, cio' spiega sia la loro estrema diffusibilità, sia l'implicazione delle KPC negli outbreaks. Nonostante la loro diffusione ad oggi esistono solo rari casi di segnalazioni di isolamenti di questo fenotipo in Italia. Scopo del presente lavoro è quello di presentare due casi clinici relativi all'isolamento di KPC nel nostro ospedale.

Le due KPC sono state isolate: la prima da una coltura di CVC in un paziente affetto da morbo di Crohn, la seconda in un campione di urina in una paziente cateterizzata colpita da Ictus. Nessun paziente aveva una storia recente di viaggi all'estero.

I ceppi sono stati caratterizzati mediante Vitek 2 ed E-test (bioMérieux) (figura I). Il fenotipo è stato confermato mediante l'esecuzione del saggio di Hodge (6) (figura I) e PCR blaKPC Gli ampliconi ottenuti sono stati in seguito sequenziati usando i seguenti primers: per la regione proteica da aa 89-399 F-5'CGGAACCATTCGCTAA-
ACTC3' e R- 3'GGCGGCGTTATCACTGTATT5'; per la regione proteica da aa 372 - $874 \mathrm{~F}-5$ 'CGCCGTGCAATACAGTGATA3' e R-3'CGTTGACGCCCAATCC5' (8). Le sequenze sono state analizzate mediante ABI Prism 310 (Applied Biosystems), e comparate con la banca dati National Center for Biotechnology Information database (http://www.ncbi.nlm.nih.gov/). La relazione genetica fra gli isolati è stata determinata mediante Rep-PCR usando il sistema DiversiLab (bioMérieux) (figura II).

Entrambi gli isolati sono risultati resistenti alle beta-lattamine, ai chinolonici, all'ertapenem, ma sensibili ad imipenem, meropenem, tigeciclina e colistina. Il test di Hodge è risultato positivo (figura I) ed il sequenziamento ha evidenziato il genotipo KPC $2(7,8)$.

I due isolati (indicati nella figura II con i numeri 1 e 2) mostravano relazione clonale. Entrambi i pazienti sono stati trattati con successo con tigeciclina. Nel presente studio presentiamo anche le farmaco sensibilità ai principali antimicrobici di altri isolati di $K$. pneumoniae. In totale abbiamo caratterizzato $149 \mathrm{~K}$. pneumoniae (dal Dicembre 2008 ad oggi) di cui 7 esibivano un comportamento KPC-like.

Delle sette sospette KPC (totale 4 pazienti, escludendo i duplicati) solo due si sono confermate con fenotipo KPC. Nella figura III sono illustrate le farmaco sensibilità globali in cui si evidenzia anche una ridotta sensibilità alla tigeciclina. Nella figura IV sono illustrate le farmaco sensibilità solo dei 4 isolati KPC-like. Nella figura V le farmaco sensibilità delle due KPC.

\section{Corresponding author: Marco Favaro}

Clinical Microbiology Laboratory, Foundation of Policlinic Tor Vergata - viale Oxford 8I 00133 Roma - Tel. 0620902073 - Fax 0620902 I59 E-mail: favaro@uniroma2.it 


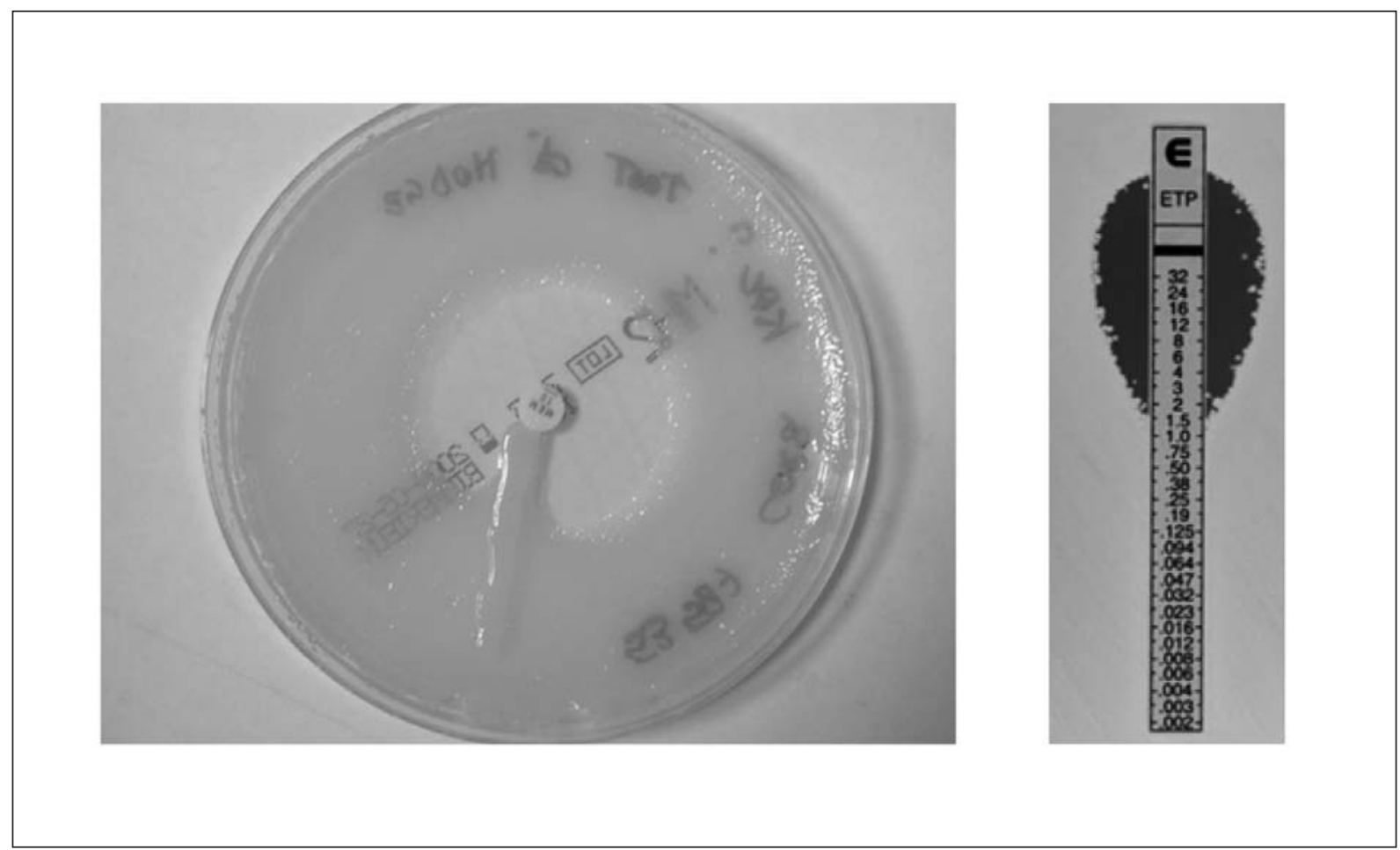

Figura I. Test di Hodge ed E-test

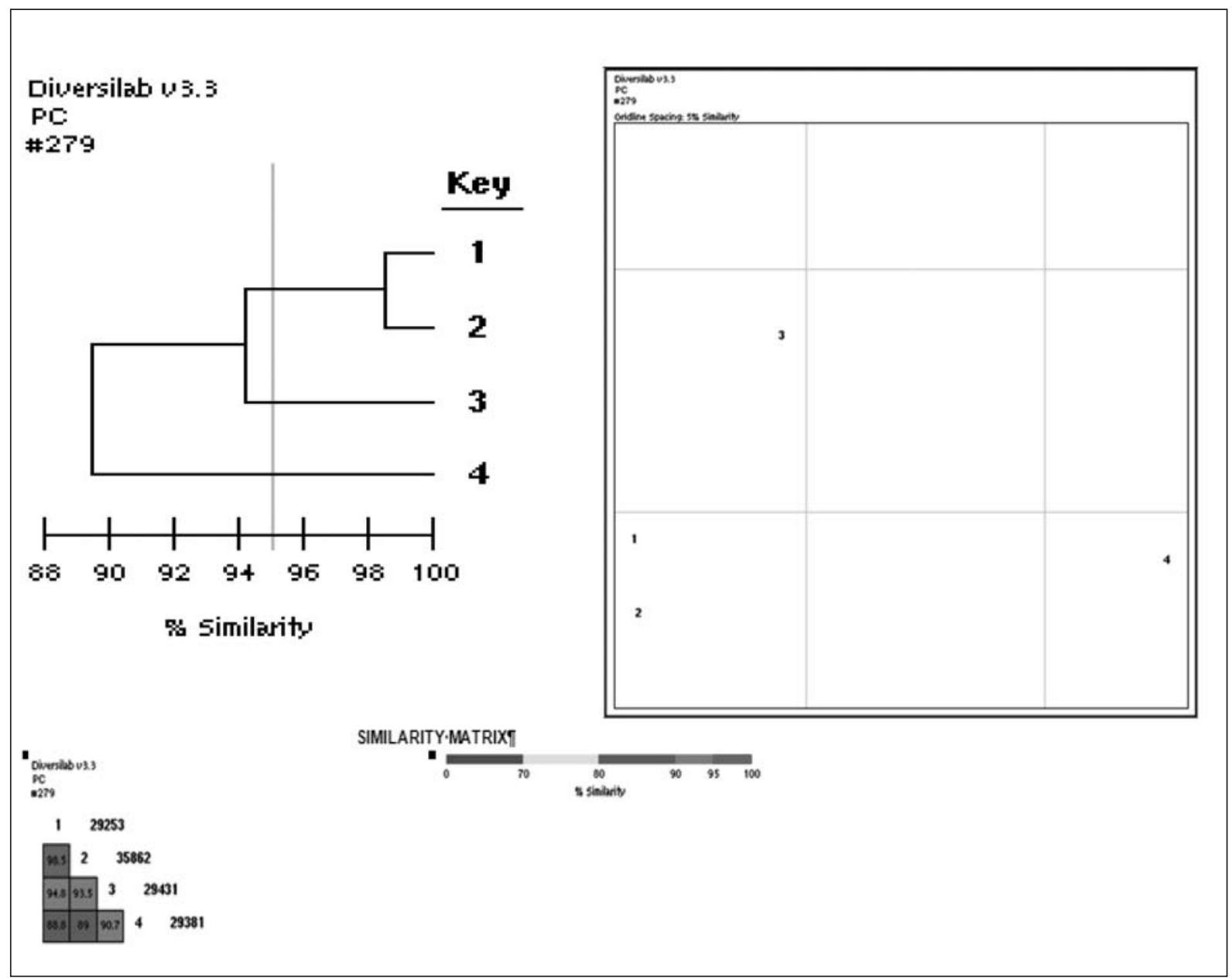

Figura II. Fingerprinting degli isolati 


\begin{tabular}{|c|c|c|c|c|c|c|c|c|c|c|}
\hline \multicolumn{11}{|c|}{ Mic:Distribuzione frequenze su 149 isolati } \\
\hline Antibiotici & 0.25 & 0.5 & 1 & 2 & 4 & 8 & 16 & 32 & 64 & 128 \\
\hline Cefepime & & & 99 & 5 & 2 & 3 & 6 & 8 & 26 & \\
\hline Cefotetan & & & & 88 & 56 & & 2 & 1 & 2 & \\
\hline Cetazidime & & & 97 & 1 & & & 8 & 2 & 41 & \\
\hline Ertapenem & & 57 & 88 & & 1 & 3 & & & & \\
\hline Imipenem & & & 154 & 4 & & & & & & \\
\hline Meropenem & 102 & 33 & 10 & 1 & 3 & & & & & \\
\hline Ciprofloxacina & 86 & 2 & 3 & & 57 & & & & 1 & \\
\hline Pip+ TPZ & & & & & 85 & 25 & & 8 & 11 & 20 \\
\hline Tigeciclina (21) & & 3 & 8 & 6 & 2 & 2 & & & & \\
\hline
\end{tabular}

Figura III. Farmaco sensibilità globale dei I 49 isolati

\begin{tabular}{|c|c|c|c|c|c|c|c|c|c|}
\hline \multicolumn{10}{|c|}{ Mic:Distribuzione frequenze } \\
\hline Antibiotici & 0.5 & 1 & 2 & 4 & 8 & 16 & 32 & 64 & 128 \\
\hline Cefepime & & & & & & & & 4 & \\
\hline Cefotetan & & & & & & 2 & 1 & 1 & \\
\hline Cetazidime & & & & & & & & 4 & \\
\hline Ertapenem & & & & 1 & 3 & & & & \\
\hline Imipenem & & & 4 & & & & & & \\
\hline Meropenem & & 4 & & & & & & & \\
\hline Levofloxacina & & & & & 4 & & & & \\
\hline Pip+TPZ & & & & & & & & & 4 \\
\hline Tigeciclina & & 3 & 1 & & & & & & \\
\hline
\end{tabular}

Figura IV. Farmaco sensibilità dei 4 isolati KPC - like

\begin{tabular}{|c|c|c|c|c|c|c|c|c|c|c|c|c|c|c|c|c|}
\hline \multicolumn{17}{|l|}{$\mathrm{MICs}(\mathrm{mg} / \mathrm{ml})$} \\
\hline Isolati & AMP & AMC & PIP & PTZ & CAZ & CTX & FEP & IPM & MEM & EPT & AMK & CIP & TGC & CS & TMP-SMX & NT \\
\hline Isolato caso I & 32 & 32 & 128 & 128 & 16 & 64 & 16 & I & 2 & 8 & 2 & 8 & $\mathrm{I}$ & 0.5 & 320 & 512 \\
\hline Isolato caso 2 & 32 & 32 & 128 & 128 & 16 & 64 & 16 & $\mathrm{I}$ & 2 & 8 & 2 & 8 & $\mathrm{I}$ & 0.5 & 320 & 512 \\
\hline
\end{tabular}

Figura V. Farmaco sensibilità delle due KPC

Legenda: AMP, ampicillina, AMC, amoxycillin-clavulanate; PIP, piperacillina, PTZ, piperacillina-tzobactam; CAZ, ceftazidime; CTX, cefotaxime, FEP, cefepime; IPM, imipenem; MEM, meropenem; EPT, ertapenem;AMK, amikacina; CIP, ciprofloxacina;TGC, tigecycline; CS, colistina; TMP-SMX, trimethoprim-sulfamethoxazole; NT, nitrofurantoina.

Le KPC rappresentano un vero dilemma per il clinico che vede ridotte le possibilità terapeutiche, ma lo sono ancor di più per il laboratorio in quanto la loro rapida e corretta identificazione è essenziale per contrastarne la diffusione. 


\section{BIBLIOGRAFIA}

1. Queenan AM, Bush K. Carbapenemases: the versatile beta-lactamases: Clin Microbiol Rev 2007, 20:440-58.

2. CLSI (Performance Standards for antimicrobial susceptibility testing): Clinical and Laboratory Standards Institute: Performance standards for antimicrobial susceptibility testing. Sixteenth informational supplement, M100-S19. 2009, Clinical and Laboratory Standards Institute, Wayne, PA

3. Evaluation of methods to identify the Klebsiella pneumoniae carbapenemase in Enterobacteriaceae: J Clin Microbiol 2007, 45:2723-5

Hossain A, Ferraro MJ, Pino RM, Dew RB II I, Moland ES, Lockhart TJ, Thomson KS, Goering RV, and Hanson ND:

4. Plasmid mediated carbapenem-hydrolyzing enzyme KPC-2 in an Enterobacter sp. Antimicrob Agents Chemother 2004,48:4438-4440.
5. Goldfarb D, Harvey SB, Jessamine K, Jessamine P, Toye B, Desjardins M: Detection of plasmid mediated KPC-Producing Klebsiella pneumoniae in Ottawa, Canada: Evidence of Intra-Hospital Transmission. J Clin Microbiol 2009, 47(6):1920-2

6. Anderson KF, Lonsway DR, Rasheed JK, Biddle J, Jensen B, McDougal LK, Carey RB, Thompson A, Stocker S, Limbago B, Patel JB. Bratu S, Landman D, Alam M, Tolentino E, and Quale J:Detection of KPC carbapenem-hydrolyzing enzymes in Enterobacter spp. From Brooklyn, New York. Antimicrob Agents Chemother 2005, 49:776-778.

7. Poirel L, Pitout JD, Nordmann P: Carbapenemases: molecular diversity and clinical consequences. Future Microbiol 2007, 2:501-12

8. Naas T, Cuzon G, Villegas MV, Lartigue MF, Quinn JP, Nordmann P: Genetic structures at the origin of acquisition of the beta-lactamase blaKPC gene. Antimicrob Agents Chemother 2008, 52(4):1257-63. 